\title{
Aplicación de técnicas estadísticas y geoestadísticas para elaborar cartografía de precipitaciones. Departamentos del occidente de Nicaragua
}

Adalila Molina Membreño y Ana de Jesús Reyes Zavala

\section{Resumen:}

Una de las grandes necesidades que se presenta en Nicaragua en los últimos años, es la de estudios, donde intervengan variables climáticas, sobre uso del suelo para las diferentes actividades que en él se practica, en La Región del Pacifico de Nicaragua, interesa hacer este tipo de análisis, ya que es aquí, donde se ubica la mayor concentración poblacional del territorio nacional, por ésta Región atraviesa la cordillera volcánica, lo que ayuda a tener un suelo muy fértil y con grandes extensiones de planicie, utilizada para el cultivo de la Caña de azúcar, maní, ajonjolí, granos básicos, frutas, etc. Por lo que se hace necesario realizar estudios, donde intervengan las variables climáticas; precipitación y temperatura y analizar el comportamiento continuo de éstas en el territorio. El Instituto Nicaragüense de Estudios Territoriales (INETER), proporcionó la información de las variable precipitación y temperatura mensual y anual para el periodo de 1954 al 2009, con las observaciones discretas de 28 estaciones (precipitación) y de 3 estaciones para medir temperatura (muy pocas para hacer interpolación), localizadas en diferentes puntos de los Departamentos de Chinandega, León y Managua, ubicados en el occidente de Nicaragua, la cantidad de observatorios proporcionados es poco densa y después de homogenizar los datos y completar donde se no se tiene información, se quedan reducida a 18 estaciones (Precipitación) y el periodo 1984-2005. En este trabajo aplicamos; los Métodos Estadísticos que permiten obtener un análisis de la relación entre las variables climáticas y otras variables, mediante los Modelos de Regresión, analizamos la precipitación, como variable dependiente y las variables independientes; latitud, longitud y elevación (usando las curvas de nivel) y un análisis de Serie Temporales para una estación meteorológica ubicada en Chinandega y analizar 
el comportamiento de la variable precipitación a lo largo del tiempo. Se realizaron diferentes cartografías continuas de precipitación promedio mensual para el periodo 1984-2005, aplicando el método de interpolación IDW y se analiza la calidad de las diferentes cartografías continuas.Palabras clave: Ciencia y Tecnologías de la Información Geográfica; Ordenamiento Territorial; Formación Universitaria.

Palabras claves: Métodos Estadísticos, Geo estadísticos, métodos de interpolación, cartografía, Modelos Lineales.

\section{Abstract:}

One of the great needs that occurs in Nicaragua in recent years, is the study, which involved climatic, land use for different activities it is practiced in the Pacific Region of Nicaragua, interested in doing this type of analysis, as it is here, where there is the greatest concentration of population of the country, for this region through the volcanic mountain range, which helps to have a very fertile soil and large expanses of plain, used for the cultivation of sugar cane, peanuts, sesame seeds, grains, fruits, etc.. As necessary studies, which involved the climatic variables, precipitation and temperature, and continuously analyzing the behavior of these in the territory. The Nicaraguan Institute of Territorial Studies (INETER) provided information of varying precipitation and monthly and annual temperature for the period 1954 to 2009, with the discrete observations of 28 stations (precipitation) and 3 stations to measure temperature (very few for interpolation), located in different parts of the departments of Chinandega, León and Managua, located in western Nicaragua, the number of observatories provided is sparse and after homogenization and complete data where no information is available, it are reduced to 18 stations (precipitation) and the period 1984-2005. In this paper we apply, the Statistical Methods that allow a discussion of the relationship between climatic variables and other variables, using Regression Models, analyze the precipitation, as the dependent variable and the independent variables, latitude, longitude and elevation (using the contour) and a Temporary Serial analysis for a meteorological station located in Chinandega and analyze the behavior of the variable precipitation over time. There have been various continuous mappings average monthly precipitation for the period 1984-2005, using the IDW interpolation method and analyze the quality of the different continuous maps. 
Keywords: Statistical Methods, IDW interpolation methods, mapping, Linear Models.

Adalila Molina Membreño (lilam7@gmail.com); Ana de Jesús Reyes Zavala (anaj_58@yahoo.es).CSIG. Facultad de Ciencias y Tecnología. Universidad Nacional Autónoma de Nicaragua-León. 


\section{INTRODUCCIÓN}

La demanda de cartografías climáticas por parte de múltiples disciplinas encuentra una serie de problemas relacionados con la cobertura espacial de los datos. La deficiente cobertura espacial de la información climática, relacionada con el carácter puntual de los datos y la escasa densidad de observatorios existentes es una limitación importante, en ocasiones determinante, para la puesta en marcha de investigaciones o la toma de decisiones ligadas a la ordenación agrícola y ambiental (Serrano y Saz, 2002).

La comunidad científica ha venido dándole un interés primordial a la modelización geo estadística de fenómenos, construyendo nuevas estructuras espacio-temporal, válida para un mayor tratamiento en muchas disciplinas como; medioambiente, epidemiologia, geología, medicina o geofísica en el estudio de la variabilidad espacial y espacio temporal. Gran parte de este esfuerzo viene encaminado en la búsqueda de nuevas herramientas que permiten estudiar fenómenos donde se incluya interacciones entre el espacio y tiempo para estudiar la variabilidad de un fenómeno de una forma separada (Alvarado, 2011).

Nicaragua por su ubicación en el continente y sus características físico geográficas, es frecuentemente afectada por diferentes fenómenos meteorológicos extremos y climáticos, los cuales causan trastornos y daños a la población, al igual que a la economía del país, es catalogado como un país potencialmente agrícola (cerca del $21 \%$ del total del área nacional tiene vocación agropecuaria), en donde la mayor parte de su economía está basada en la agricultura, la cual participa en un $33 \%$ del total del Producto Interno Bruto, de ella depende el desarrollo económico que el país necesita para satisfacer las necesidades básicas de su población. Sin embargo, la productividad agrícola está en dependencia del comportamiento del régimen de precipitación. Se han hecho estudios para elaborar y analizar las cartografías a nivel nacional por ejemplo sobre el Tema la Sequía. (INIDE Atlas Cenagro 2001).

La Región del Pacifico de Nicaragua, lugar del estudio, se ubican los Dptos. de Chinandega, León y Managua en los cuales está la mayor concentración poblacional del territorio nacional, por esta Región atraviesa la cordillera volcánica, lo que ayuda a tener un suelo muy fértil y con grandes extensiones de planicie, utilizada para el cultivo de la Caña de azúcar, maní, ajonjolí, granos básicos, 
frutas, etc. Por lo que se hace necesario realizar estudios, donde intervengan las variables climáticas; precipitación y temperatura, para analizar el comportamiento continuo de éstas en el territorio. El Instituto Nicaragüense de Estudios Territoriales (INETER),proporcionó la información de la variable precipitación promedio anual y temperatura promedio mensual para el periodo de 1954 al 2009.

El objetivo de esta investigación, es aplicar Técnicas Estadísticas y de Estadística espaciales para analizar y comparar cartografías sobre el comportamiento de la precipitación promedio mensual, a partir de la información obtenida de las 18 estaciones pluviométricas ubicadas en los departamentos de Chinandega, León y Managua.

Utilizando el software Estadístico SPSS, construimos los modelos de Regresión Lineal Simple y Múltiple y se busca un modelo de mejor ajuste, para la variable dependiente Precipitación y como independientes; elevación, latitud y longitud. Se realizó el análisis de Series Temporales, para analizar el comportamiento de la precipitación promedio mensual a lo largo del periodo 1984-2005 solamente para un observatorio, localizado en el Municipio de Chinandega, por ser este el que contiene la información más completa.

Se realizó el análisis Geo estadístico con el uso del software ArcGIS.10 como herramienta de los Sistemas de Información Geográfico (SIG), para analizar y comparar las cartografías temáticas, sobre el comportamiento continuo en el espacio geográfico de la precipitación promedio mensual registrada en el periodo 1984-2005, a partir de los 18 puntos discretos en el territorio, por medio del método de Interpolación; IDW, obteniendo información de forma espacial en lugares donde no se tiene información sobre la precipitación. Dada la poca cantidad de información en los observatorios con que se cuenta para medir la precipitación en el territorio nacional, es que se hace necesaria la aplicación de estas técnicas geo estadísticas. 


\section{AREA DE ESTUDIO}

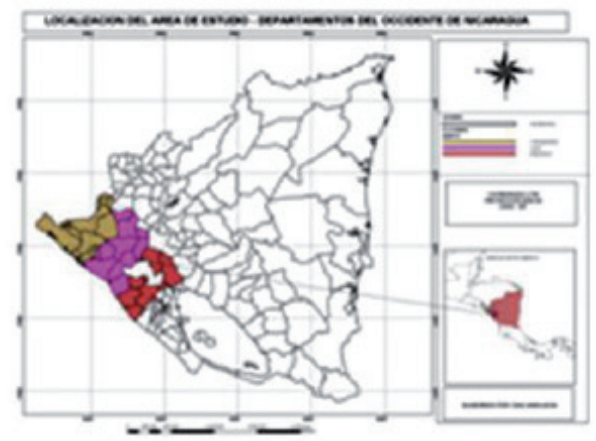

Figura 1: Mapa de Localización del área de estudio

La República de Nicaragua tiene una extensión de $130,370 \mathrm{~km}^{2}$, de los cuales el $92.0 \%$ es tierra y el restante $8.0 \%$ es agua. Es el país con mayor extensión territorial de Centroamérica y está ubicado en el centro geográfico del istmo. El $42.8 \%$ de la tierra tiene vocación agrícola, mientras el $25.9 \%$ es forestal. El país se divide en 15 departamentos y dos regiones autónomas, las que a su vez comprenden 153 municipios. Tiene fronteras con Honduras y Costa Rica, así como costas en el océano Pacífico y en el mar Caribe (BCIE, BID y Banco Mundial, 2011).

Nicaragua se encuentra la mayor parte del año, bajo la influencia de los vientos Alisios, provenientes de los anticiclones subtropicales de las Azores y Bermudas. Estos vientos son constantes, de poca variabilidad y tienen la particularidad de arrastrar masas de aire húmedo del mar Caribe hacia el interior de Nicaragua. Este viento cálido y húmedo penetra por la vertiente del Atlántico hacia la vertiente del Pacífico, ejerciendo un efecto importante sobre el estado del tiempo y el clima del país. Las temperaturas medias anuales presentan variaciones relativamente pequeñas, mientras que los valores extremos diarios muestran oscilaciones considerables. La variabilidad estacional del régimen térmico, se aprecia en las vertientes de los principales sistemas montañosos del país, en particular entre los 200 y 900 metros sobre el nivel medio del mar (INETER (2000).

El Departamento de Chinandega, se caracteriza por presentar una fisiografía conformada por planicies, lomeríos, serranías, conos volcánicos y zonas costeras de estuarios. Las mayores elevaciones las constituyen el Volcán San Cristóbal $(1,745 \mathrm{msnm})$, el Casita $(1,405 \mathrm{msnm})$ y el Chonco $(1,105 \mathrm{msnm})$, presentando pen- 
dientes muy escarpadas en las faldas (mayor de 50\%) y ondulada a fuertemente ondulada en el pie de monte (15 a 50\%). El clima del departamento de Chinandega se caracteriza por ser cálido en las zonas bajas y más frescas y agradables en las Zonas de mayor altura (conos volcánicos). De acuerdo a la clasificación climática de Köppen el departamento es definido como tropical de sabana. Presenta una marcada estación seca que va de noviembre a abril y el período lluvioso comprende de mayo a octubre. Las temperaturas medias se hallan entre los 21 y $30^{\circ} \mathrm{C}$ y las máximas hasta los $42^{\circ} \mathrm{C}$. La precipitación anual máxima alcanza $2,000 \mathrm{~mm}$ y la mínima hasta $500 \mathrm{~mm}$ anuales en algunos municipios. De acuerdo a la clasificación realizada por el proyecto hidrológico centroamericano, el territorio del departamento de Chinandega cuenta con cuatro cuencas hidrográficas bien definidas, siendo éstas: Las cuencas Río Negro, Estero Real, ríos entre el Estero Real y el Volcán Cosigüina y ríos entre el Volcán Cosigüina y el Tamarindo, de las cuales dos de ellas (Estero Real y Ríos entre el Volcán Cosigüina y Tamarindo) comparten áreas potenciales de riego con el departamento de León (INIDE, 2012).

El departamento de León se caracteriza por presentar una fisiografía conformada por planicies, lomeríos, serranías, conos volcánicos y zonas costeras de estuarios, las cuales se describen en función de la forma y origen de todos los accidentes geográficos que caracterizan el departamento, es el tercero de mayor superficie de los siete que comprenden la Macro Región. (INIDE 2012).

El clima en el departamento de Managua se caracteriza por ser de sabana tropical con una prolongada estación seca y temperaturas que oscilan entre los $27.5^{\circ} \mathrm{C}$ y $28^{\circ} \mathrm{C}$, la precipitación media anual varía entre los 1,000 y $1,500 \mathrm{~mm}$, a excepción del municipio de El Crucero, que tiene una variación de temperatura promedio de $22^{\circ} \mathrm{C}$ y $28^{\circ} \mathrm{C}$, siendo éste, uno de los pocos lugares de la costa del pacifico en poseer estas temperaturas. Presenta topografía inclinada hacia la costa del lago o banda norte, o una zona montañosa o de alturas que comprende: Las sierras de Managua, las sierritas de Santo Domingo y la península de Chiltepe. En cuanto a recursos hídricos se refiere, el municipio de Managua cuenta con una serie de lagunas de origen volcánico como: la laguna de Asososca, Tiscapa y Xiloá (INIDE 2012). 


\section{MATERIAL Y MÉTODO}

Fuente de la información estadística y geográfica: Instituto Nacional de Estudios Territoriales (INETER) 1954 al 2009.

Lugares de estudio: departamentos de Nicaragua; Chinandega, León y Managua.

Variables utilizadas: precipitación, elevación, longitud y latitud.

Modelo Digital de Elevación: Mapas de Chinandega, León y Managua:

Programa utilizados: para procesamiento y análisis estadístico; SPSS y R y ArcGIS.10 para la realización de las cartografías temáticas.

Las Bases de datos fueron proporcionadas, por el Instituto Nicaragüense de Estudios Territoriales (INETER), en archivos Excel y pdf. con información; de las coordenadas de ubicación de los observatorios que miden precipitación y temperatura, de los tres departamentos incluidos en el estudio, información sobre la precipitación de 28 observatorios y de temperatura solamente para 3 estaciones ubicadas en Corinto, Chinandega y Aeropuerto Godoy en la ciudad de León, dicha información está registrada desde 1954 al 2009.

Antes de hacer todo el análisis se procedió a homogenizar la información, organizarla y depurarla en Excel, todo ese proceso generó reducción de los observatorios y años a ser analizados, debido a que en algunos observatorios había un número considerable de datos omitidos para la variable precipitación, en aquellos casos que se tenían menos datos faltantes se estimaron y en los que no se tenían registros para muchos meses y años no se tomaron en cuenta para el análisis, después de este proceso, se quedan reducida a 18 estaciones (Precipitación) y el periodo de 1984-2005. Seguidamente la base de datos se analiza desde el punto de vista estadístico en el software SPSS (Statistical Package for the Social Sciences) y R y el análisis espacial con ArcGIS.10.

\section{Análisis aplicados}

En este trabajo se realizaron los Análisis Estadísticos y Análisis Espacial para la variable; Precipitación, en promedios mensuales en el periodo 1984-2005. 
En el Análisis Estadístico; Primeramente se realizó el Análisis Exploratorio de la variable precipitación encontrándose observaciones atípicas de precipitación. Los gráficos de Dispersión de la variable Precipitación vs elevación, precipitación vs altitud y precipitación vs longitud, en ninguno de los casos se observa ninguna tendencia. De igual manera se procedió a realizar un Análisis de regresión lineal con el proceso por pasos en el programa SPSS con el fin de obtener un modelo matemático, que nos fuera útil para la realización de la técnica de interpolación global. El primer modelo de Regresión Lineal simple, incluye la variable precipitación como variable dependiente y como independiente la longitud, un segundo modelo se hace con precipitación y latitud y un tercer modelo con precipitación y elevación, observándose los resultados del análisis que existe una relación entre estas variables incluidas en el modelo con la precipitación. Se continuó haciendo los modelos de Regresión múltiple, donde se incluyen dos variables independientes, después tres variables independientes, se continuo el proceso, buscando aumentar el $R^{2}$ (es el que nos da una explicación buena o mala del modelo), pero el $R^{2}$ no mejoro (menos del 0.30). Por tanto, concluimos que el modelo de Regresión no es un buen predictor y es más conveniente aplicar el modelo de interpolación mediante el Kriging. Antes de aplicarlo, también se debe verificar si los datos siguen una distribución normal, encontrándose que los datos de precipitación no tienen una distribución normal, lo cual puede afectar a la estimación de la precipitación por el método de Kriging y no aplicamos este método, aplicamos el método IDW, que no requiere de estos supuestos. Villegas (2011).

Se realiza la Interpolación espacial, los métodos de interpolación basados en modelos de regresión simple o múltiple permiten estimar el valor de la variable climática a partir de una serie de variables independientes. Los métodos más utilizados en climatología son los basados en técnicas geo estadísticas, entre estos están: el Kriging y el Inverso de la Distancia (IDW). Las estimaciones realizadas mediante los diferentes métodos de Kriging se basan en una suma ponderada de las $n$ observaciones más cercanas al punto de estimación. El peso de cada uno de los puntos será recogido para que la estimación no se encuentre sesgada y tenga la menor varianza posible en relación a otras posibles combinaciones lineales entre el conjunto de observaciones disponibles. El método de interpolación de Inverso de la Distancia, requiere la selección de un área o un número de puntos mínimos para la predicción, los valores estimados se obtienen con los puntos de muestreo existentes. Los puntos más cercanos tendrán un peso superior, ya que existe una ponderación en función de la distancia (Vicente y Saz, 2002). 
En el Análisis Espacial; Para la elaboración de las cartografías temáticas se realizan los siguientes pasos: 1. Revisar Las coordenadas de las estaciones meteorológicas, en este caso están en unidades UTM, el esferoide es de WGS84 y se establece la proyección correspondiente. 2 . Antes de ser usada la información en SIG, se realizó el proceso de conversión del formato con extensión .xls a formato shapefile con extensión .shp en el software de ArcGis.10 se adicionaron los puntos discretos (observatorios) en el mapa de los departamentos de Chinandega, León y Managua, obteniendo como primer resultado; el mapa de Localización de los observatorios de precipitación, ubicados en los tres departamentos. (Fig. No.1).

Se aplica la técnica espacial IDW, para valores de $r=1, r=2$ y $r=4$, dando como resultado los modelos geográficos que permiten analizar el comportamiento continuo de la variable precipitación mensual en el periodo 1984-2005, a partir de los 18 observatorios (datos discretos) en los Departamentos de Chinandega, León y Managua. Para la realización y análisis de estos modelos se consultó el tutorial (ESRI 2012), (FRANZ 2011) y (Suárez M. Astudillo C. 2011). La última parte del trabajo consiste en la edición de esos mapas para su presentación en papel o impresión.

\section{Interpolación a partir del método de la distancia inversa (IDW).}

La interpolación del punto problema se realiza asignando pesos a los datos del entorno en función inversa de la distancia que los separa -inverse distance weighting, IDW-.

$$
\hat{z}_{j}=\sum_{i=1}^{n} k_{i j} \cdot z_{i}
$$

donde $Z_{j}$ es el valor estimado para el punto j; $n$ es el número de puntos usados en la interpolación; $z_{i}$ el valor en el punto i-ésimo y $K_{i j}$ el peso asociado al dato i en el cálculo del nodo j. los pesos k varían entre 0 y 1 para cada dato y la suma total de ellos es la unidad. La interpolación mediante Distancia Inversa Ponderada, determina los valores de celda a través de una combinación ponderada linealmente de un conjunto de puntos de muestra. La ponderación es una función de la distancia inversa. La superficie que se interpola debe ser la de una variable dependiente (Precipitación) de la ubicación.

El método de la distancia inversa (IDW) se apoya en el concepto de continuidad espacial, con valores más parecidos para posiciones cercanas que se van 
diferenciando conforme se incrementa la distancia. El uso de este algoritmo ha sido empleado en la representación de variables con continuidad espacial, como las isócronas, los mapas de pendientes y orientaciones a partir de la altitud. La distribución de la precipitación no es continua en el espacio, y no está sujeta a ninguna gradación, ecuación o parámetro que regule su disposición. La aplicación del método de interpolación permite generar resultados cartográficos y estadísticos nuevos, que son predictivos e irreales. Pero el proceso de abstracción que supone la generación de un mapa a partir de este tipo de datos puede ser válido desde el punto de vista cartográfico, aumentando la legibilidad y claridad de la variable representada. Los mapas de isolíneas son por tanto una alternativa de expresión cartográfica para la distribución de la precipitación. (Felicísimo Capitulo1).

Para presentar un ejemplo de aplicación de las Series de Tiempo, modelar y analizar el comportamiento de la variable precipitación media mensual, se escogió un observatorio ubicado en el municipio de Chinandega (el que tiene mayor información).

\section{RESULTADOS Y ANÁLISIS}

\section{Resultados Estadísticos}

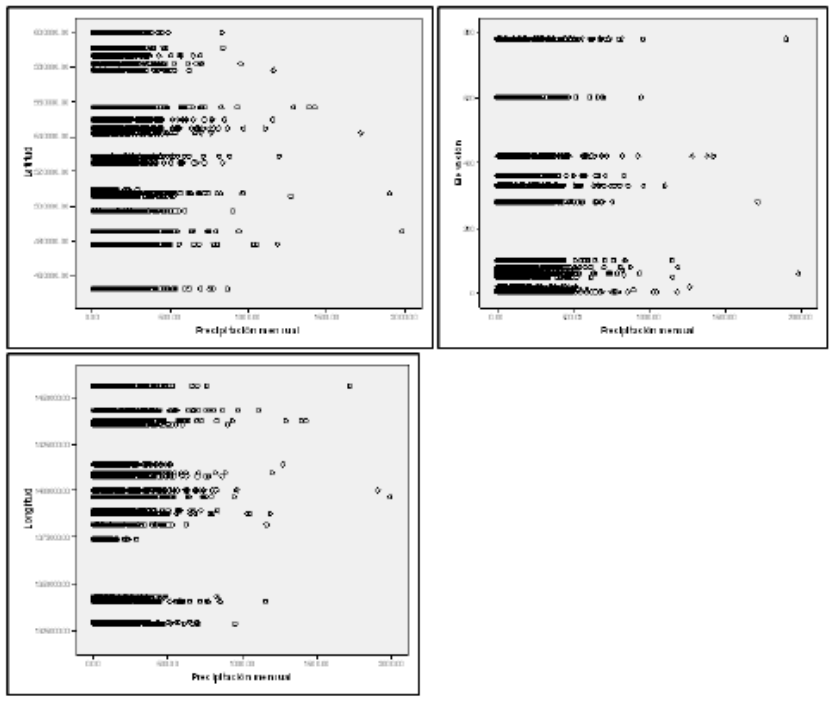

Figura 2: Gráficos de dispersión. 1) Precipitación vs latitud. 2) Precipitación vs elevación. 3) Precipitación vs longitud. 
Se puede apreciar en estos gráficos de dispersión, que no existe una relación clara relación entre la variable precipitación media mensual y latitud, longitud, pero con elevación sí.

\section{Análisis de Series Temporales}

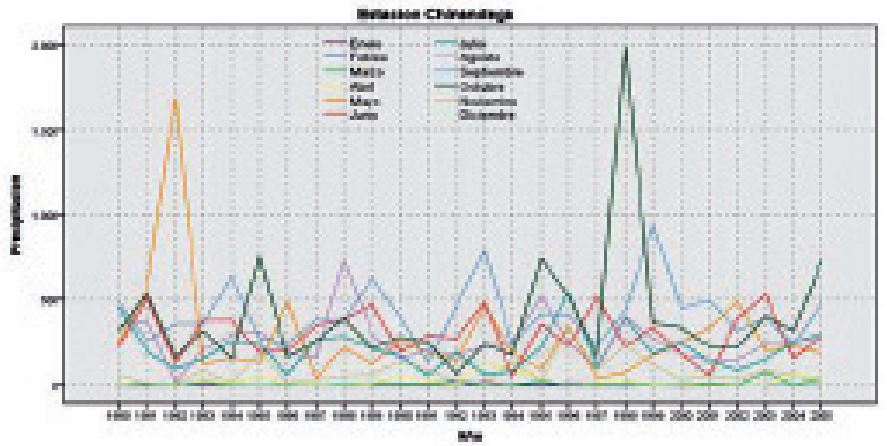

Figura. 3: Gráfico de Línea

Se ve en esta gráfica, definidas las líneas por meses, un comportamiento que no excede de los 1000 milímetros cúbicos de agua en los meses de lluvia (de mayo a octubre), a través de los años, a excepción de mayo de 1982 y octubre de 1998.

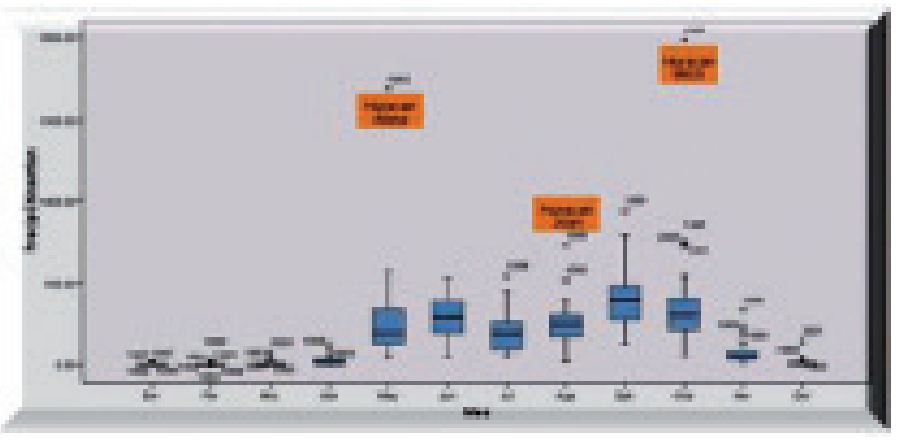

En la gráfica de caja se observa que; para mayo del 82 y octubre del 98 , en estos meses y años se alcanzaron las mayores precipitaciones, valores de precipitación atípicos, no se observa simetría en la distribución. 


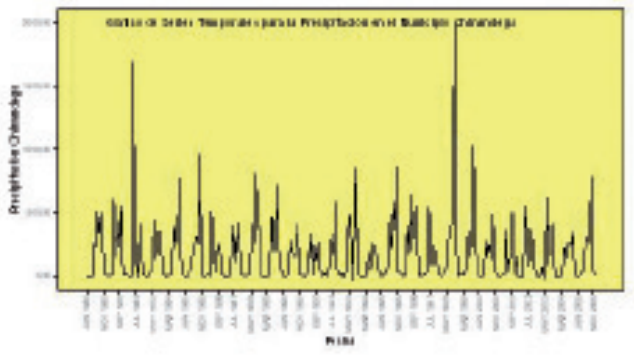

Figura 5: Grafica de Series de Tiempo

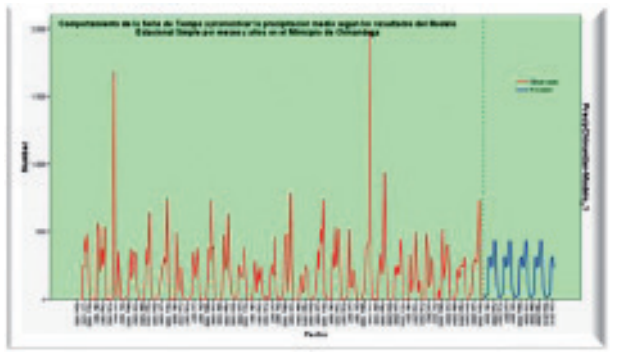

Figura 6: Modelo de predicción

Se observa en este gráfico de series de tiempo, que en los invierno de 1982 y 1998 altas precipitaciones en el pacifico de Nicaragua. Con el Modelo Estacional Simple, al hacer el pronóstico para 5 años se observa un comportamiento estacional. Al revisar el por qué, se tiene como información adicional, que esto es debido a los huracanes que se produjeron en esos años Alleta y Mitch respectivamente, los cuales registraron precipitaciones entre los 1500 y los 2000 milímetros cúbicos de agua. 


\section{Resultados Geográficos de Interpolación IDW}

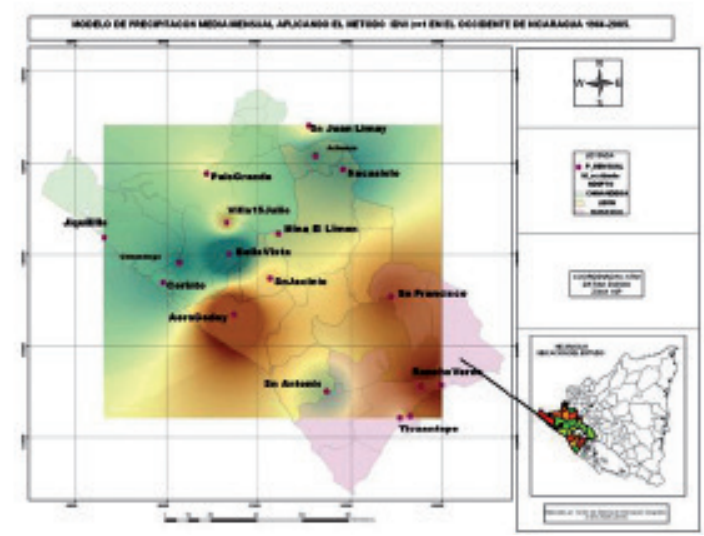

Figura 7 Mapa de Interpolación IDW para r=1

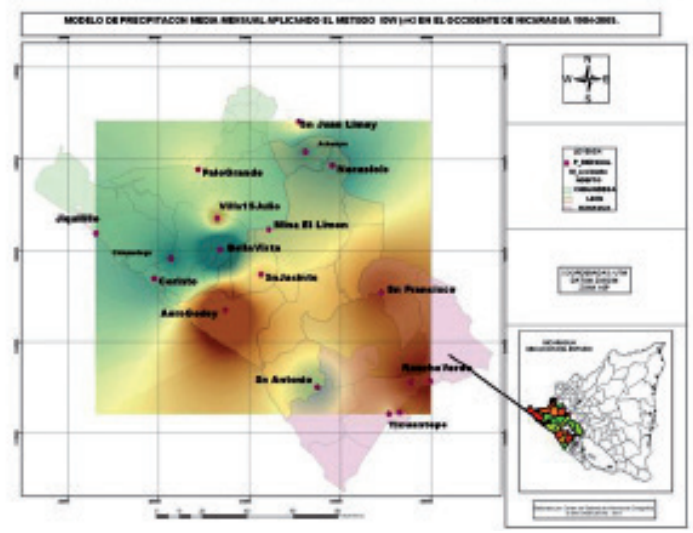

Figura 8 Mapa de Interpolación IDW para r=2 


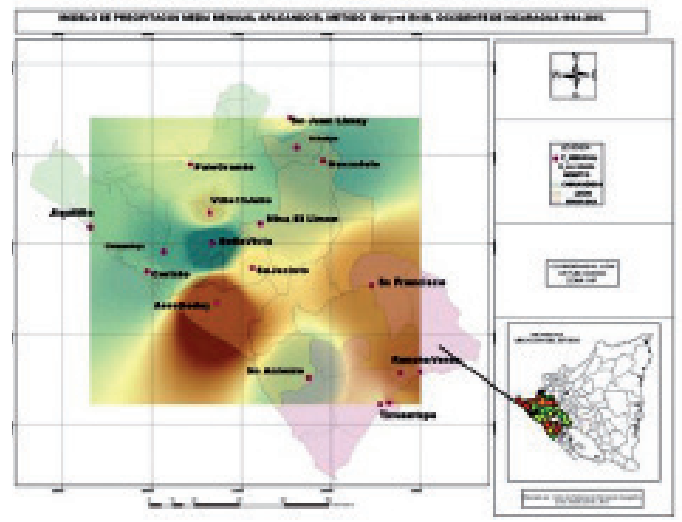

Figura 9 Mapa de Interpolación IDW para r=4

Las cartografías obtenidas de los modelos de interpolación IDW para distintos valores de $r$ aumentan la legibilidad y claridad de la variable Precipitación representada en el espacio a medida que aumenta dicho valor, los valores están considerado en tres categorías de precipitación, representada en degradación de colores que va de marron a azul, así los lugares con azul más fuertes indican territorios con altas precipitaciones y los de peores resultados se observan en marron. Por otro lado se ve la tendencia de la precipitación, que va en la dirección oeste hacia el sur este. Se observan altos valores de precipitación en las estaciones localizadas en las partes altas y con mayor vegetación.

\section{CONCLUSIÓN}

Se pudo apreciar una cantidad mayor de observatorios de precipitación y escasos estaciones de temperaturas. Lo cual dificulto la posibilidad de poder hacer la aplicación de interpolación espacial y por tanto no conseguir las cartografías para la variable temperatura. Por otro lado los grandes vacios en los observatorios y años para la variable precipitación, causo eliminación de mucha información, esto afecta para poder continuar haciendo análisis mediante otros métodos y por tanto hacer comparaciones .

Con la aplicación de los métodos estadísticos se logra obtener resultados importantes para buscar causas que pueden estar incidiendo sobre la situación de 
no normalidad de los datos, la ocurrencia de dos huracanes muy intensos en ese periodo, hace que se presenten datos atípicos, los datos estén sesgados, se observa el comportamiento de la serie que presenta picos altos en esos dos momentos, estos análisis son necesarios para decidir que método geo estadísticos de análisis es más conveniente aplicar, e ir viendo como se complementan para explicar el comportamiento de la variable precipitación media mensual y reforzando sus resultados para el análisis.

Estos mapas de interpolación son por tanto una alternativa de expresión cartográfica para la distribución de la precipitación en el espacio. La elaboración de este tipo de trabajo para los Departamentos de occidentes, permiten hacer el mejor uso de las cartografías espaciales de representación, lo que servirá para mejorar la planificación de las actividades agrícolas relacionadas con el factor climático.

El uso de las técnicas estadísticas y geo estadísticas, Idw, mostraron una forma para concebir el análisis de la información espacial en zonas de difícil acceso y con pocos puntos discretos.

\section{BIBLIOGRAFIA}

- Villegas (2011). Análisis geo estadístico con ArcGIS parte 2. Análisis exploratorio de los datos [consulta:12-03-201].Disponible http://www.aguaysig. com/2011/03/analisis-geoestadistico-con-arcgis.html

- Suárez M. Astudillo C. 2011 Desarrollo del módulo ISOLINEAS en ArcGis. Aplicación cálculo de isoyetas en el estado de Guerrero, Instituto Mexicano de Tecnología del Agua, Jiutepec, Mor., México. Disponibles en http://www.ommac. org/congres02011/document/extenso/Ext2011014.pdf

- ESRI (2012). Geostatistical Analyst Tutorial

- FRANZ (2011). Crear un mapa de isoyetas e isotermas en ArcGIS [consulta: 03-04-2013]. Disponible en http://www.acolita.com/wp-content/uploads/Estaciones-meteorol\%C3\%B3gicas-ArcGIS.png

- BCIE (2011) Ficha Estadística de Nicaragua. disponible en http://www.bcie. org/uploaded/content/article/1249943988.pdf (3 BCIE, BID y Banco Mundial) 
Fichas Estadísticas de Nicaragua 04/03/13

- INIDE. Atlas Cenagro (2001) Disponible en http://www.inide.gob.ni/atlas/caracteristicasdep/Chinandega.htm (inide), (última visita 04/03/13)

- INIDE (2012). Sistema Nacional de Estadisticas Vitales (SINEVI). Disponible en http://www.inide.gob.ni/estadisticas/Cifras\%20municipales $\% 20 a \% C 3 \%$ B10\%202012\%20INIDE.pdf (última visita 04/03/13)

- Regionalizacion Biofisica para el Desarrollo Agropecuario Departamento de León 1999, Disponible en http://cedoc.magfor.gob.ni/documentos/cedoc/B100192.pdf (última visita 04/03/13)

- INETER (2000) Atlas Climátimatica 1971-2000 disponible en http://webserver2. ineter.gob.ni/Direcciones/meteorologia/clima\%20nic/caracteristicasdelclima. html visita 18/02/13

- Alvarado Welman Rosa 2011. Revista de Matemática: Teoría y Aplicaciones 18(2) : 325-342 cimpa - ucr issn: 1409-2433).

- Felicísimo A. Capitulo1. Conceptos básicos, modelos y simulación disponibles http://www6.uniovi.es/ feli/CursoMDT/Tema1/Page32.html). Marzo 2013 